\title{
Socio-Demographic, Clinical Correlates and Predictors of Adverse Outcome in Substance Use Disorder (SUD) Patients Attending the De-Addiction Clinic of a Tertiary Care Hospital in Eastern India
}

\author{
Dr Sikha Mukhopadhyay, MBBS, MD \\ Associate Professor, Department of Psychiatry, Institute of Psychiatry, IPGME \& R, Kolkata \\ Dr Parthasarathy Biswas, MBBS, MD \\ Assistant Professor, Institute of Psychiatry, IPGME \& R, Kolkata
}

\section{CONFLICT OF INTEREST : None}

\section{ABSTRACT}

\begin{abstract}
Substance Use Disorder (SUD) is a serious public health problem in India. The present study was planned to study the socio-demographic, clinical correlates and predictors of adverse outcomes in all new patients attending the De-addiction clinic in a tertiary hospital in Eastern India. All new patients visiting the De-addiction clinic were assessed and interviewed. Four groups were made viz Alcohol, Opioid, Cannabinoid and Other SUDs. A total of 200 patients were included in the study, of whom majority were males. A positive family history of substance use was evident in $44.1 \%$ of the patients in the Cannabinoid group. The mean duration of use was $180.87 \pm 95.64$ for the alcohol group, $78.70 \pm 66.41$ for the opioid group, $94.0 \pm 85.70$ for the Cannabinoid group, $104.2 \pm 79.37$ for other SUDs. The most common substance used was alcohol $(n=117)$. Our findings suggest that the initiation of substance use occurs during late teen age years and early $20 \mathrm{~s}$ in case of Opioid and Cannabinoid group and in case of Alcohol group in their early 30s. In our study, poor social support correlated with family complications, marital complications and social complications. Motivation clearly and significantly correlated with financial, family, marital and social complications suggesting that motivation to leave alcohol/drugs is influenced by these factors. The motivation level influenced follow-up rates with poor to superficial motivation predicting poorer follow up rates. On the other hand good and fair motivation correlated with increased adherence to attending Group therapy. Motivation to leave drugs/alcohol correlated with drop-out \& relapse rates with poorer outcomes in form of relapses and drop out related to poor motivation to leave drugs. Financial security in form of employment is critical for adherence to treatment and follow-up rates which in turn influences recovery and rehabilitation. Motivation is cornerstone to relapses and management of motivation enhancement and adherence to treatment can reduce relapses.
\end{abstract}

Key Words : Substance use Disorders (SUDs), relapse rate, dropout rate, socio-demographic risk factors

Corresponding Author ${ }^{\circ}$

Dr Parthasarathy Biswas, Assistant Professor

Institute of Psychiatry, IPGME \& R

Kolkata - 700025

Phone : 9836778063

Email : ps.bs69@gmail.com

\section{INTRODUCTION}

Substance use disorders (SUDs) have been the focus of various medical specialties especially amongst psychiatrists. SUDs may be viewed as one 
of the epidemic diseases which not only affects the individual in form of greater health care costs but also extends to the family and the society at large thus leading to greater economic burden on the country. A meta-analysis by Reddy and Chandrashekharan (1998) revealed an overall substance use prevalence of $6.9 / 1000$ for India with urban and rural rates being 5.8 and 7.3/1000 population. The National Household Survey of Drug Use in the country (Ray R 2004) was the first systematic effort to document the National prevalence of drug use which found that most common substance of use was Alcohol (21.4\%) followed by cannabis $(3.0 \%)$ and opioids (0.7\%). The Drug Abuse Monitoring System (Ray 2004) which evaluated the primary substance of abuse in various Government deaddiction centres found that the major substance were alcohol $(43.9 \%)$, opioids $(26 \%)$ and cannabis (11.6\%). Abuse of legally prescribed drugs is also increasingly becoming alarming. Therefore SUDs is being recognized as a significant public health problem in India.

Research has yet to explain fully the physiological and psychological processes leading to drug abuse. Family stress, lack of coping skills, peer pressure, personality disorder, comorbid psychiatric illnesses, social stress and free availability of these substances of abuse act as risk factors (Sadock \& Sadock 2000). It has been seen that $75 \%$ of dependent individuals start drug/alcohol use before 20 years of age which suggests that the loss in terms of human potential is incalculable (Kumar SM 2011). Children and Adolescent SUDs is one of the major areas of concern in India (Bansal \& Banerjee 1993; Tripathi \& Lal 1999; Benegal, Satyaprakash Nagaraja 2008).

SUDs have been viewed as a chronic disorder in which relapse and remission is the part of the natural course of the disorder (Annual report MSJ \& FW). Relapse is considered when a person returns to the previous pattern of substance abuse that was established prior to abstinence. The corner stone of any SUDs management regimen is Relapse prevention. The goals of treatment are abstinence from/reduction of use of substance, frequency and severity of relapses and improvement of psychological and social adaptive functioning (Murthy P 2008). The course and outcome of substance abuse disorders is poor due to non-utilization of treatment services beyond a period of one month emphasizing the need to retain patients in follow-up (Murthy 2008). Higher income and duration of in-patient treatment were predictors of improved outcome at 3 months followup. Drop-out rates ranged from $10-40 \%$.

To get the society rid of this epidemic one needs to understand the socio-demographic \& clinical profile risk factors leading to SUDs and explore what the positive predictors of outcome are. The present study is therefore an effort to find the patterns of substance use disorders, psychiatric and physical comorbidities, relapse rates, motivation level, follow-up rates, with various socio-demographic factors and treatment related issues.

\section{PATIENT AND METHODOLOGY}

The Ethics committee approval was obtained from the Institutional Ethics Committee of IPGME \& R, Kolkata, India prior to commencement of this study. The Institute has a well established De-addiction Clinic with active and efficient referral system from General psychiatry as well as from other specialties and super-specialty departments of this tertiary care hospital in Eastern India. The specialty clinic has both in-patient and out-patient departments specifically for de-addiction purposes. The patients were approached individually and explained about the objectives of the study in their preferred language and written consent was taken from the patients willing to participate in the study. Willing patients were then inducted into the study.

We conducted an observational study with crosssectional design with follow-up after 3 months. All consecutive new patients visiting the clinic during the period February 2011 to July 2013 were taken up for the study. The predesigned and pretested Sociodemographic profile sheet and Clinical profile sheet was used to assess the patients. Socio-demographic data were determined with questions about gender, marital status, age, religion, occupational status, 
educational status, residential status, family income and individual income. Clinical examination and assessments were done separately by two qualified Consultant Psychiatrists of this Institute. Assessment of physical comorbidities including investigations were assessed by the specialty clinics to which they were refereed to or referred from. Previous treatment records acted as secondary data. ICD-10 DCR criteria for drug/alcohol dependence were used for the study. A total of 200 patients who gave consent to the study were included in the study. The Addiction Severity Index (ASI) scale $5^{\text {th }}$ edition validated by UNODC (2010) was used to assess the severity of substance abuse. The department's mental state examination was used by the Consultant Psychiatrists to assess the psychiatric comorbidities. The family history of psychiatric and SUDs was assessed by interviewing the significant family care givers of the patient. All patients were referred to Group therapy sessions conducted by trained social workers and clinical psychologists.

\section{STATISTICAL ANALYSIS}

The data thus generated was tabulated and analyzed using SPSS 14.0 software (Chicago, Illinois). The socio-demographicprofiles of the various groups were compared by chi-square tests. ANOVA was used to compare the continuous variables like age, age of onset, family income, duration of use and duration of dependence, number of follow-ups etc. Bonferrinipost-hoc tests were used to see the differences across the groups. Then, univariate analysis was performed to investigate the association between socio-demographic variables with substance use disorders and other clinical variables like relapses, number of hospitalizations, number of follow-ups and whether they attended group therapy or not. Next, the associations that were significant in the univariate analysis were analyzed using logistic regression models. The dependent variable in logistic regression was alcohol/drug dependence syndrome groups. Those who were following after 3-months were reanalyzed using the above mentioned pattern.

\section{RESULTSAND OBSERVATIONS}

A total number of new cases (n) were 200 consecutive new patients visiting the Deaddiction Clinic in this Institute during the study period. Patients were predominately males 197 (98.5\%) with females accounting for only $3(1.5 \%)$ of total cases.

The Opioid group $(n=34)$ consisted of 23 patients meeting ICD-10 DCR criteria of dependence on Heroin and 11 patients of other forms or opioids like Dextropropoxyphene, Natural Opium etc. The Other SUDs group consisted primarily of Solvent/ inhalant dependence patients, Benzodiazepine dependence and Nicotine dependence syndrome as primary substance of abuse/dependence. The Alcohol Group, Cannabinoid group and Other SUDs consisted of 117, 34 and 15 patients respectively.

Majority of patients were Hindu by religionand the study had a male preponderance. Majority belonged to the 20-49 year age group. Urban preponderance was seen in the Opioid group (82\%) and Other SUDs (e.g. Volatile Solvent) [73\%] unlike other groups where the distribution was more even. Significant majority of patients in the Alcohol Group (Group 1) were married and were employed at time of participation in the trial. Majority of patients came from a $80 \mathrm{~km}$ radius of the institute. Majority (50-67\%) of referrals to De-addiction clinic came from other departments of the Institute.

Primary substance of use was predominantly Alcohol dependence syndrome $(\mathrm{N}=117)$, followed by Opioid group $(\mathrm{N}=34)$ which included 20 patients dependent on heroin and 14 patients dependent on other opioids. The SUD others Group consisted of 8 patients dependent on sedative and hypnotics, 4 patients dependent on inhalants/solvents and 3 patients were dependent primarily on nicotine. In the Alcohol group the most common secondary substance of dependencewastobacco $(75.2 \%)$.

Approximately $50-67 \%$ of patients took previous treatment for their SUD or SUD related problems prior to visiting the Deaddiction clinic of this Institute. Approximately $25-40 \%$ of patients were 
hospitalized prior to visiting the Deaddiction clinic of this Institute. The Alcohol group had significantly more comorbid physical illnesses than other 3 groups $(\mathrm{p}<0.001)$. The Cannabinoid group (Group III) had significantly more comorbid Schizophrenia and schizophrenia like psychotic disorders where as Alcohol group (Group II) had significantly greater dual diagnosis of Bipolar Disorders and other affective disorders. The Duration of use (in months) shows that there was significant difference across groups with Alcohol group patients starting the initiation approximately 15 years prior to coming to the clinic. The duration of dependence i.e. the time when patient first fulfilled the Dependence criteria as per ICD 10-DCR was also higher in the alcohol group (100.56 months $\mathrm{SD}=63.8)$. This suggests that the time from initiation to dependence was longest in alcohol group shortest in Opioid and Other SUD group (which included the Solvent/Inhalant abuse with the Cannabinoid group falling in between these extremes.

\section{Table 1 : Comparison of Socio-demographic profile among alcohol, opioid, cannabinoid and other SUDs group patients}

\begin{tabular}{|c|c|c|c|c|c|}
\hline Variables & $\begin{array}{l}\text { Alcohol } \\
\text { (Group1) } \\
\mathbf{N}=117 \\
\mathbf{N}(\%)\end{array}$ & $\begin{array}{c}\text { Opioid } \\
\text { (Group II) } \\
\mathbf{N}=34 \\
\mathbf{N}(\%)\end{array}$ & $\begin{array}{c}\text { Cannabinoid } \\
\text { (Group III) } \\
\mathbf{N}=34 \\
\mathbf{N}(\%)\end{array}$ & $\begin{array}{c}\text { Others } \\
\text { (Group IV) } \\
\mathbf{N}=15 \\
\mathbf{N}(\%)\end{array}$ & Significance \\
\hline $\begin{array}{l}\text { Gender } \\
\text { Male } \\
\text { Female }\end{array}$ & $\begin{array}{l}115(98.3) \\
2(1.7 \%)\end{array}$ & $\begin{array}{l}34(100) \\
-\end{array}$ & $\begin{array}{l}33(97.1) \\
1(2.9)\end{array}$ & $\begin{array}{l}15(100) \\
-\end{array}$ & $\begin{array}{l}\mathrm{NS} \\
\mathrm{df}=3\end{array}$ \\
\hline Age (Mean \pm SD) & $39.9 \pm 9.49$ & $27.55 \pm 5.58$ & $26.26 \pm 8.98$ & $30.53 \pm 12.00$ & $\begin{array}{l}F=30.62^{* * *} \\
P<0.001 ; d f=3\end{array}$ \\
\hline $\begin{array}{l}\text { Age category } \\
<14 \text { years } \\
15-19 \text { years } \\
20-49 \text { years } \\
>50 \text { years }\end{array}$ & $\begin{array}{l}- \\
- \\
95(81.2 \%) \\
22(18.8)\end{array}$ & $\begin{array}{l}- \\
2(5.9) \\
32(94.1) \\
-\end{array}$ & $\begin{array}{l}- \\
4(11.8) \\
29(85.3) \\
1(2.9)\end{array}$ & $\begin{array}{l}1(6.7) \\
- \\
13(86.7) \\
1(6.7)\end{array}$ & $\begin{array}{l}\mathrm{P}<0.001 \\
\mathrm{df}=9\end{array}$ \\
\hline $\begin{array}{l}\text { Marital stat } \\
\text { Never married } \\
\text { Married } \\
\text { Divorced/separated }\end{array}$ & $\begin{array}{l}14(12.0) \\
98(83.8) \\
5(4.3)\end{array}$ & $\begin{array}{l}19(55.9) \\
15(44.1) \\
0\end{array}$ & $\begin{array}{l}22(64.7) \\
11(32.4) \\
1(2.9)\end{array}$ & $\begin{array}{l}10(66.7) \\
4(26.7) \\
1(6.7)\end{array}$ & $\begin{array}{l}\mathrm{P}<0.001 \\
\mathrm{df}=6\end{array}$ \\
\hline $\begin{array}{l}\text { Education } \\
\text { Illiterate } \\
\text { Primary } \\
\text { Middle (upto IX) } \\
\text { Matric/higher sec } \\
\text { Grad \& post grad }\end{array}$ & $\begin{array}{l}4(3.4) \\
17(14.5) \\
37(31.6) \\
40(34.2) \\
19(16.2) \\
\end{array}$ & $\begin{array}{l}4(11.8) \\
6(17.6) \\
9(26.5) \\
14(41.2) \\
1(2.9) \\
\end{array}$ & $\begin{array}{l}1(2.9) \\
4(11.8) \\
20(58.8) \\
6(17.6) \\
3(8.8) \\
\end{array}$ & $\begin{array}{l}- \\
- \\
6(40.0) \\
5(33.3) \\
4(26.7) \\
\end{array}$ & $\begin{array}{l}\mathrm{P}<0.05 \\
\mathrm{Df}=12\end{array}$ \\
\hline $\begin{array}{l}\text { Employed } \\
\text { Never employed } \\
\text { Presently } \\
\text { unemployed } \\
\text { Employed }\end{array}$ & $\begin{array}{l}1(0.9) \\
25(21.4) \\
91(77.8)\end{array}$ & $\begin{array}{l}6(17.6) \\
8(23.5) \\
20(58.8)\end{array}$ & $\begin{array}{l}7(20.6) \\
15(44.1) \\
12(35.3)\end{array}$ & $\begin{array}{l}5(33.3) \\
3(20.0) \\
7(46.7) \\
\end{array}$ & $\begin{array}{l}<0.001 \\
\mathrm{Df}=6\end{array}$ \\
\hline $\begin{array}{l}\text { Income } \\
<1500 \mathrm{Rs} \\
1500-5999 \mathrm{Rs} \\
>6000 \mathrm{Rs}\end{array}$ & $\begin{array}{l}30(25.6) \\
39(33.3) \\
48(41.0)\end{array}$ & $\begin{array}{l}13(38.2) \\
14(41.2) \\
7(20.6)\end{array}$ & $\begin{array}{l}19(55.9) \\
13(38.2) \\
2(5.9)\end{array}$ & $\begin{array}{l}8(53.3) \\
3(20.0) \\
4(26.7)\end{array}$ & $\begin{array}{l}<0.001 \\
\mathrm{Df}=6\end{array}$ \\
\hline $\begin{array}{l}\text { Family Income } \\
\text { (Mean } \pm \text { SD) }\end{array}$ & $\begin{array}{l}9773.5 \pm \\
9035.48\end{array}$ & $\begin{array}{l}8594.11 \pm \\
6752.63\end{array}$ & $\begin{array}{l}9932.35 \pm \\
9636.58\end{array}$ & $\begin{array}{l}12800.0 \pm \\
12013.08\end{array}$ & $\begin{array}{l}F=0.962 \\
\text { NS }\end{array}$ \\
\hline
\end{tabular}


Socio-Demographic, Clinical Correlates and Predictors of Adverse Outcome in Substance Use Disorder

\begin{tabular}{|c|c|c|c|c|c|}
\hline Variables & $\begin{array}{c}\text { Alcohol } \\
\text { (Group1) } \\
\mathbf{N}=117 \\
\mathbf{N}(\%)\end{array}$ & $\begin{array}{c}\text { Opioid } \\
\text { (Group II) } \\
\mathbf{N}=34 \\
\mathbf{N}(\%)\end{array}$ & $\begin{array}{c}\text { Cannabinoid } \\
\text { (Group III) } \\
\mathbf{N}=34 \\
\mathbf{N}(\%)\end{array}$ & $\begin{array}{c}\text { Others } \\
\text { (Group IV) } \\
\mathbf{N}=15 \\
\mathbf{N}(\%)\end{array}$ & Significance \\
\hline $\begin{array}{l}\text { Religion } \\
\text { Hindu } \\
\text { Islam } \\
\text { Others }\end{array}$ & $\begin{array}{l}110(94.0) \\
4(3.4) \\
3(2.6)\end{array}$ & $\begin{array}{l}26(76.5) \\
7(20.6) \\
1(2.9)\end{array}$ & $\begin{array}{l}29(85.3) \\
5(14.7) \\
-\end{array}$ & $\begin{array}{l}14(93.3) \\
1(6.7) \\
-\end{array}$ & $\begin{array}{l}<0.05 \\
\mathrm{Df}=6\end{array}$ \\
\hline $\begin{array}{l}\text { Family Type } \\
\text { Nuclear } \\
\text { Joint } \\
\text { Extended }\end{array}$ & $\begin{array}{l}71(60.7) \\
43(36.8) \\
3(2.6)\end{array}$ & $\begin{array}{l}15(44.1) \\
18(52.9) \\
1(2.9)\end{array}$ & $\begin{array}{l}19(55.9) \\
15(44.1) \\
-\end{array}$ & $\begin{array}{l}11(73.3) \\
4(26.7) \\
-\end{array}$ & $\begin{array}{l}\mathrm{NS} \\
\mathrm{Df}=6\end{array}$ \\
\hline $\begin{array}{l}\text { Locality } \\
\text { Urban } \\
\text { Rural } \\
\text { Semiurban }\end{array}$ & $\begin{array}{l}53(45.3) \\
32(27.4) \\
32(27.4)\end{array}$ & $\begin{array}{l}28(82.4) \\
4(11.8) \\
2(5.9)\end{array}$ & $\begin{array}{l}15(44.1) \\
10(29.4) \\
9(26.5)\end{array}$ & $\begin{array}{l}11(73.3) \\
4(26.7) \\
-\end{array}$ & $\begin{array}{l}\mathrm{P}<0.01 \\
\mathrm{Df}=6\end{array}$ \\
\hline $\begin{array}{l}\text { Social Status } \\
\text { Upper SES } \\
\text { Lower SES } \\
\text { Poor } \\
\text { Below Poverty line } \\
\end{array}$ & $\begin{array}{l}56(47.9) \\
38(55.9) \\
19(16.2) \\
4(3.4) \\
\end{array}$ & $\begin{array}{l}12(35.3) \\
15(44.1) \\
4(11.8) \\
3(8.8) \\
\end{array}$ & $\begin{array}{l}10(29.4) \\
12(35.3) \\
8(23.5) \\
4(11.8) \\
\end{array}$ & $\begin{array}{l}8(53.3) \\
3(20.0) \\
4(26.7) \\
-\end{array}$ & $\begin{array}{l}\mathrm{NS} \\
\mathrm{Df}=9\end{array}$ \\
\hline $\begin{array}{l}\text { Distance } \\
\text { Local } \\
\text { Upto } 40 \mathrm{~km} \\
40-80 \mathrm{~km} \\
80-160 \mathrm{~km} \\
>160 \mathrm{~km}\end{array}$ & $\begin{array}{l}8(6.8) \\
38(32.5) \\
46(39.3) \\
19(16.2) \\
6(5.1)\end{array}$ & $\begin{array}{l}7(20.6) \\
11(32.4) \\
13(38.2) \\
3(8.8) \\
-\end{array}$ & $\begin{array}{l}3(8.8) \\
10(29.4) \\
14(41.2) \\
6(17.6) \\
1(2.9)\end{array}$ & $\begin{array}{l}2(13.3) \\
8(53.3) \\
4(26.7) \\
1(6.7) \\
-\end{array}$ & $\begin{array}{l}\text { NS } \\
\mathrm{Df}=12\end{array}$ \\
\hline $\begin{array}{l}\text { Referral } \\
\text { Self } \\
\text { Medical } \\
\text { Non-medical } \\
\text { Relative } \\
\text { IPGMER }\end{array}$ & $\begin{array}{l}11(9.4) \\
42(35.9) \\
2(1.7) \\
4(3.4) \\
58(49.6)\end{array}$ & $\begin{array}{l}1(2.9) \\
9(26.5) \\
- \\
1(2.9) \\
23(67.6)\end{array}$ & $\begin{array}{l}- \\
12(35.3) \\
- \\
4(11.8) \\
18(52.9)\end{array}$ & $\begin{array}{l}3(20.0) \\
3(20.0) \\
- \\
- \\
9(60.0)\end{array}$ & $\begin{array}{l}\mathrm{NS} \\
\mathrm{Df}=12\end{array}$ \\
\hline
\end{tabular}

Table 2 : Comparison of Clinical profile among alcohol, opioid, cannabinoid and other SUDs group patients

\begin{tabular}{|c|c|c|c|c|c|}
\hline Variables & $\begin{array}{c}\text { Alcohol } \\
\text { (Group1) } \\
\text { N=117 } \\
\mathbf{N}(\%)\end{array}$ & $\begin{array}{l}\text { Opioid } \\
\text { (Group II) } \\
\begin{array}{c}\mathrm{N}=34 \\
\mathrm{~N}(\%)\end{array}\end{array}$ & $\begin{array}{c}\text { Cannabinoid } \\
\text { (Group III) } \\
\text { N=34 } \\
\text { N(\%) }\end{array}$ & $\begin{array}{l}\text { Others } \\
\text { (Group IV) } \\
\begin{array}{c}\text { N=15 } \\
\text { N(\%) }\end{array}\end{array}$ & Significance \\
\hline $\begin{array}{l}\text { Previous treatment } \\
\text { Yes }\end{array}$ & $65(55.6)$ & $23(67.6)$ & $17(50)$ & $8(53.3)$ & $\begin{array}{l}\text { NS } \\
\mathrm{Df}=3\end{array}$ \\
\hline $\begin{array}{l}\text { Ever Hospitalized } \\
\text { Yes }\end{array}$ & $39(33.3)$ & $14(41.2)$ & $9(26.5)$ & $6(40.0)$ & $\begin{array}{l}\mathrm{NS} \\
\mathrm{Df}=3\end{array}$ \\
\hline $\begin{array}{l}\text { Medical illness } \\
\text { Yes }\end{array}$ & $56(47.9)$ & $6(17.6)$ & $5(14.7)$ & $2(13.3)$ & $\begin{array}{l}\mathrm{P}<0.001 \\
\mathrm{Df}=3\end{array}$ \\
\hline
\end{tabular}


Socio-Demographic, Clinical Correlates and Predictors of Adverse Outcome in Substance Use Disorder

\begin{tabular}{|c|c|c|c|c|c|}
\hline Variables & $\begin{array}{l}\text { Alcohol } \\
\text { (Group1) } \\
\mathrm{N}=117 \\
\mathrm{~N}(\%)\end{array}$ & $\begin{array}{l}\text { Opioid } \\
\text { (Group II) } \\
\begin{array}{l}\mathrm{N}=34 \\
\mathrm{~N}(\%)\end{array}\end{array}$ & $\begin{array}{l}\text { Cannabinoid } \\
\text { (Group III) } \\
\begin{array}{c}\text { N=34 } \\
\text { N(\%) }\end{array}\end{array}$ & $\begin{array}{l}\text { Others } \\
\text { (Group IV) } \\
\begin{array}{c}\text { N=15 } \\
\text { N(\%) }\end{array}\end{array}$ & Significance \\
\hline $\begin{array}{l}\text { Comorbid Psychiatric } \\
\text { Disorder } \\
\text { - F20-29 } \\
\text { - F30-34 } \\
\text { - F40-45 } \\
\text { - F60 } \\
\text { - ADHD/CD/ODD } \\
\text { - Substance induced } \\
\text { sychosis } \\
\end{array}$ & $\begin{array}{l}4(3.4) \\
33(28.2) \\
8(6.8) \\
6(5.1) \\
- \\
5(4.3)\end{array}$ & $\begin{array}{l}1(2.9) \\
8(23.5) \\
- \\
2(5.9) \\
- \\
3(8.8)\end{array}$ & $\begin{array}{l}8(23.5) \\
8(23.5) \\
- \\
2(5.9) \\
1(2.9) \\
6(17.6)\end{array}$ & $\begin{array}{l}1(6.7) \\
4(26.7) \\
1(6.7) \\
2(13.3) \\
1(6.7) \\
-\end{array}$ & $\begin{array}{l}\mathrm{P}<0.001 \\
\mathrm{Df}=18\end{array}$ \\
\hline $\begin{array}{l}\text { Family H/o } \\
\text { Psychiatric illness } \\
\quad \text { • Yes }\end{array}$ & $14(12.0)$ & $1(2.9)$ & $4(11.8)$ & $2(13.3)$ & $\begin{array}{l}\mathrm{NS} \\
\mathrm{Df}=3\end{array}$ \\
\hline $\begin{array}{l}\text { Family } \mathrm{H} / \mathrm{O} \\
\text { Substance abuse } \\
\qquad \text { Yes } \\
\end{array}$ & $42(35.9)$ & $5(14.7)$ & $14(44.1)$ & $2(13.3)$ & $\begin{array}{l}\mathrm{P}<0.05 \\
\mathrm{Df}=3\end{array}$ \\
\hline $\begin{array}{l}\text { Duration of Use } \\
\text { (months) Mean } \pm \text { SD }\end{array}$ & $180.87 \pm 95.64$ & $78.70 \pm 66.41$ & $94.0 \pm 85.70$ & $104.2 \pm 79.37$ & $\begin{array}{l}\mathrm{F}=17.53^{* * *} \\
\mathrm{Df}=3\end{array}$ \\
\hline $\begin{array}{l}\text { Duration of } \\
\text { dependence (months) } \\
\text { Mean } \pm \text { SD }\end{array}$ & $100.56 \pm 63.8$ & $62.38 \pm 48.84$ & $63.64 \pm 57.12$ & $91.86 \pm 68.67$ & $\begin{array}{l}F=5.502^{* *} \\
P<0.01 \\
D f=3\end{array}$ \\
\hline
\end{tabular}

Table 3 : Comparison of Addiction Severity Index variables (ASI) of alcohol, opioid, Cannabinoid and other SUDs group patients

\begin{tabular}{|l|c|l|l|l|l|}
\hline \multicolumn{1}{|c|}{ Variables } & $\begin{array}{c}\text { Alcohol } \\
\text { (Group1) } \\
\text { N = 117 } \\
\text { N (\%) }\end{array}$ & $\begin{array}{c}\text { Opioid } \\
\text { (Group II) } \\
\mathbf{N = 3 4} \\
\mathbf{N ~ ( \% )}\end{array}$ & $\begin{array}{c}\text { Cannabinoid } \\
\text { (Group III) } \\
\mathbf{N}=34 \\
\mathbf{N}(\%)\end{array}$ & $\begin{array}{c}\text { Others } \\
\text { (Group IV) } \\
\mathbf{N}=15 \\
\mathbf{N}(\%)\end{array}$ & Significance \\
\hline Health Complications & & & & & \\
Nil & $19(16.2)$ & $4(11.8)$ & $8(23.5)$ & $6(40.0)$ & NS \\
Mild & $50(42.7)$ & $16(46.1)$ & $17(50.0)$ & $5(33.3)$ & Df=9 \\
Moderate & $36(30.8)$ & $9(26.5)$ & $7(20.6)$ & $4(26.7)$ & \\
Severe & $12(10.3)$ & $5(14.7)$ & $2(5.9)$ & - & \\
\hline Financial complication & & & & & \\
Nil & $12(10.3)$ & $4(11.8)$ & $4(11.8)$ & $7(46.7)$ & P<0.05 \\
Mild & $27(23.1)$ & $6(17.6)$ & $10(29.4)$ & $2(13.3)$ & Df=9 \\
Moderate & $55(47.0)$ & $18(52.9)$ & $10(29.4)$ & $5(33.3)$ & \\
Severe & $23(19.7)$ & $6(17.6)$ & $10(29.4)$ & $1(6.7)$ & \\
\hline
\end{tabular}




\begin{tabular}{|c|c|c|c|c|c|}
\hline Variables & $\begin{array}{c}\text { Alcohol } \\
\text { (Group1) } \\
\text { N = 117 } \\
\text { N (\%) }\end{array}$ & $\begin{array}{c}\text { Opioid } \\
\text { (Group II) } \\
\text { N = 34 } \\
\mathbf{N}(\%)\end{array}$ & $\begin{array}{c}\text { Cannabinoid } \\
\text { (Group III) } \\
\text { N = 34 } \\
\text { N (\%) }\end{array}$ & $\begin{array}{c}\text { Others } \\
\text { (Group IV) } \\
\text { N = 15 } \\
\mathbf{N}(\%)\end{array}$ & Significance \\
\hline $\begin{array}{l}\text { Occupation complication } \\
\text { Nil } \\
\text { Mi wld } \\
\text { Moderate } \\
\text { Severe }\end{array}$ & $\begin{array}{l}19(16.2) \\
42(35.9) \\
36(30.8) \\
20(17.1)\end{array}$ & $\begin{array}{l}7(20.6) \\
8(23.5) \\
12(35.3) \\
7(20.6)\end{array}$ & $\begin{array}{l}5(14.7) \\
15(44.1) \\
6(17.6) \\
8(23.5)\end{array}$ & $\begin{array}{l}4(26.7) \\
4(26.7) \\
5(33.3) \\
2(13.3)\end{array}$ & $\begin{array}{l}\mathrm{NS} \\
\mathrm{Df}=9\end{array}$ \\
\hline $\begin{array}{l}\text { Legal complications } \\
\text { Nil } \\
\text { Mild } \\
\text { Moderate } \\
\text { Severe }\end{array}$ & $\begin{array}{l}87(74.4) \\
20(17.1) \\
7(6.0) \\
3(2.6)\end{array}$ & $\begin{array}{l}28(82.4) \\
3(8.8) \\
2(5.9) \\
1(2.9)\end{array}$ & $\begin{array}{l}23(67.6) \\
5(14.7) \\
5(14.7) \\
1(2.9)\end{array}$ & $\begin{array}{l}11(73.3) \\
1(6.7) \\
1(6.7) \\
2(13.3)\end{array}$ & $\begin{array}{l}\text { NS } \\
\text { Df }=9\end{array}$ \\
\hline $\begin{array}{l}\text { Marital complications } \\
\text { Nil } \\
\text { Mild } \\
\text { Moderate } \\
\text { Severe }\end{array}$ & $\begin{array}{l}32(27.4) \\
63(53.8) \\
15(12.8) \\
7(6.0)\end{array}$ & $\begin{array}{lr}20 & (58.8) \\
12 & (35.3) \\
2 & (5.9) \\
- & \\
\end{array}$ & $\begin{array}{l}24(70.6) \\
9(26.5) \\
- \\
1(2.9)\end{array}$ & $\begin{array}{l}12(80.0) \\
2(13.3) \\
- \\
1(6.7)\end{array}$ & $\begin{array}{l}\mathrm{p}<0.001 \\
\mathrm{Df}=9\end{array}$ \\
\hline $\begin{array}{l}\text { Social complications } \\
\text { Nil } \\
\text { Mild } \\
\text { Moderate } \\
\text { Severe }\end{array}$ & $\begin{array}{l}25(21.4) \\
50(42.7) \\
40(34.2) \\
2(1.7)\end{array}$ & $\begin{array}{l}4(11.8) \\
8(23.5) \\
18(52.9) \\
4(11.8)\end{array}$ & $\begin{array}{l}4(11.8) \\
14(41.2) \\
13(38.2) \\
3(8.8)\end{array}$ & $\begin{array}{l}8(53.3) \\
2(13.3) \\
4(26.7) \\
1(6.7)\end{array}$ & $\begin{array}{l}\mathrm{P}<0.01 \\
\mathrm{Df}=9\end{array}$ \\
\hline
\end{tabular}

On the variables of Addiction Severity Index higher financial and marital complications occurred in Alcohol and Opioids group while social complications were more in Opioid and Cannabinoid group. Opioid group had more severe psychosocial complications due to drug abuse or dependence. Cannabinoid group had high family history of SUD $(44.1 \%)$ as compared to other groups $\left(10.86^{*} \mathrm{p}<0.05\right)$. However, no significant difference was seen across groups on variable of Family history of Psychiatric Disorders. In Pre-morbid personality, although there was no differences across groups howeverComorbid Cluster ' $\mathrm{B}$ 'Personality disorders were seen in $26.5 \%$ of cases in opioid group. The study however could not find any increased chance of any group having any particular type of personality disorder.

Motivation to leave alcohol/drugs is an important indicator of repeated relapses and adverse outcome in cases of SUDs. Cannabinoid group had poorer motivation (32.4\%) to leave drugs than other groups though the difference was non-significant. Another $50 \%$ of patients had superficial motivation.

Social support is an important factor in determination of outcome and treatment compliance as well as follow-ups and adherence to treatment. On comparison of Social Support no difference was found across groups with $80-95 \%$ patients having fair to good social support. This suggests that patients coming to our clinic had better social support. Also Indian patients like other groups of psychiatric disorders have a better outcome due to better social support.

\section{TREATMENT VARIABLES}

Cannabinoid group had higher dual diagnosis of Psychotic disorders whereas Alcohol group had 
higher comorbidity of BPAD. Of the 117 patients in the alcohol group, 53 patients were prescribed some aversive medications as maintenance therapy. About $68 \%$ patients were on Acamprosate, $22 \%$ on Disulfiram, and around $7 \%$ were on Baclofen. Out the 34 patients in the Opioid group a total of 14 patients were on Naltrexone alone and 1 patient was prescribed combination of Naloxone and Buprenorphine. About $62 \%$ of the Cannabinoid group was prescribed antipsychotic medications probably due to the increased comorbidity of Schizophrenia and increased chances of cannabis induced nonaffective psychosis. The Alcohol group patients were on Antidepressants or Mood stabilizers possibly due to the higher comorbidity of affective disorders in this group as compared to the other groups. Most common form of detoxification of alcohol group was with help of benzodiazepines and in the Opioid group by Clonidine and NSAIDS.Therefore most of the cases were started on conventional treatments that included withdrawal of the drug, management of the withdrawal symptoms and management of co-morbidities. The only $40 \%$ of the Opioid group patients gave consent for Group Psychotherapy and continued with the group sessions. On the contrary around $62 \%$ and $53 \%$ of the Alcohol group and Other SUDs attended the sessions.

\section{FOLLOW UP RATES \& DROP - OUT/RELAPSE RATES}

The differences in the follow-up rates across the groups were not significant. The mean follow up rates for the Alcohol group was $3.15(\mathrm{SD}=2.58)$, Opioid group was $3.17(\mathrm{SD}=3.50)$, Cannabinoid group was $4.32(\mathrm{SD}=4.68)$, and Other SUDs was 3.26 $(\mathrm{SD}=3.75)$.

\section{Table 4 : Comparison of Drop-out rates and relapse rates of alcohol, opioid, Cannabinoid and other SUDs group patients}

\begin{tabular}{|l|c|c|c|c|l|}
\hline \multirow{7}{*}{ Variables } & $\begin{array}{c}\text { Alcohol } \\
\text { (Group1) }\end{array}$ & $\begin{array}{c}\text { Opioid } \\
\text { (Group II) }\end{array}$ & $\begin{array}{c}\text { Cannabinoid } \\
\text { (Group III) }\end{array}$ & $\begin{array}{c}\text { Others } \\
\text { (Group IV) }\end{array}$ & Significance \\
& $\mathbf{N}=117$ & $\mathbf{N}=34$ & $\mathbf{N}=34$ & $\mathbf{N}=15$ & \\
\hline Drop out/Relapsed & $\mathbf{N}(\%)$ & $\mathbf{N}(\%)$ & $\mathbf{N}(\%)$ & $\mathbf{N}(\%)$ & \\
Follow up & $66(56.4)$ & $21(61.8)$ & $20(58.8)$ & $10(66.7)$ & Df=3 \\
& $51(43.6)$ & $13(38.2)$ & $14(41.2)$ & $5(33.3)$ & NS \\
\hline
\end{tabular}

The drop-out and relapse rates ranged from $56.4 \%$ in Alcohol group to $66.7 \%$ in the Other SUDs group. These differences were however not significant. Yet the $56-66 \%$ drop out rate may be considered high across all groups.

\section{CORRELATION ANALYSIS}

Age at presentation correlated positively with Marital Status (Spearman's Rho $0.586 \mathrm{p}<0.001$ ), Employment status (Spearman's Rho 0.459 $\mathrm{p}<0.001$ ), Income of patient (Spearman's Rho 0.425 $\mathrm{p}<0.001$ ) and Social Status (Spearman's Rho 0.586 $\mathrm{p}<0.001)$. With increasing age there is increased Medical Illness (Spearman's Rho 0.395; p<0.001) suggesting that long duration of SUD caused various medical complications to develop. Age at presentation correlated with increase in Marital
Complication (Spearman's Rho 0.413; p<0.001) and Social complications (Spearman's Rho 0.146; $\mathrm{p}<0.001)$ which meant that marital partners and the society at large had difficulty in coping with long duration of the illness. However those patients who were employed (Spearman's Rho $0.333 \mathrm{p}<0.001$ ) and were in the higher income (Spearman's Rho 0.250; $\mathrm{p}<0.001)$ category were at a better chance of getting a life partner. Also those who got married were more likely to have well adjusted premorbid personality. But over the years these patients who did get the chance to get a life partner faced more marital complications (Spearman's Rho 0.705; $\mathrm{p}<0.001$ ).

Educational level correlated with greater social support (Spearman's Rho 0.169; p<0.05). Higher income correlated positively with social status. Higher income correlated positively with increased 
Medical illness suggesting extravagant life style leads to drug/substance abuse as well as various life style medical illnesses. Income negatively correlated with Financial complications (Spearman's Rho -0.199 $\mathrm{p}<0.01$ ), family complications (Spearman Rho -0.277 $\mathrm{p}<0.001$ ) and social complications (Spearman's Rho $-0.018 \mathrm{p}<0.05)$. Family income positively correlated with duration of dependence suggesting that patients may have used their family resources to sustain their drug/alcohol abuse. Poor social status meant greater financial complications (Spearman's Rho 0.223; $\mathrm{p}<0.01)$. Comorbid Medical illness has a negative impact on marital status of these patients. Family history of substance abuse correlated with social supporti.e. positive family history of substance abuse led to poor social support (Spearman's Rho 0.206; $\mathrm{p}<0.01$ ). Positive family history of psychiatric illness in first degree relatives correlated with Marital problems (Spearman's Rho 0.193; $\mathrm{p}<0.01$ ) possibly because it was difficult for life partners to deal with both their spouses alcohol/drug abuse and family's burden to deal with psychiatric illness in another family member.

Poor social support correlated with family complications (Spearman's Rho -0.236 p<0.01), marital complications (Spearman's Rho -0.148 $\mathrm{p}<0.01$ ) and social complications (Spearman's Rho $-0.206 \mathrm{p}<0.01$ ). Motivation clearly and significantly correlated with financial, family, marital and social complications suggesting that Motivation to leave alcohol/drugs is influenced by these factors. The motivation level influenced follow-up rates (Spearman's Rho $0.375 \mathrm{p}<0.001$ ) with poor to superficial motivation predicting a poorer follow up rates. On the other hand good and fair motivation correlated with increased adherence to attending Group therapy (Spearman's Rho 0.495; $\mathrm{p}<0.001$ ). Motivation to leave drugs/alcohol correlated with drop-out \& relapse rates with poorer outcomes in form of relapses and drop out related to poor motivation to leave drugs.

\section{DISCUSSION}

India is facing an epidemic in form of substance use disorders (SUDs). In the present study, majority of new patients $(98.5 \%)$ who attended the De-addiction clinic of this institute were males. While females are largely confined to indoors, the males have more easy access to illicit drugs and alcohol. Moreover, the embarrassment and shame one might face on revealing this behavior to family and society at large prevents females abusing drugs/alcohol leads to poor health seeking. Other reports from Asia have also reported a male preponderance (Nessa et al 2008). Majority of patients were Hindu by religion but national survey found no difference in religion (Kumar SM 2011).

There was a significant difference across the groups regarding age at presentation to the clinic with Alcohol group having a mean age of almost 40 years where as the Opioid and Cannabinoid group patients had a mean age (at presentation) in their mid-20s. Educational status and employment status of patients have varied across studies. In the present study, $70 \%$ of the Cannabinoid group could not study beyond middle school. Almost $50 \%$ of the Alcohol group and 'Other SUDs' were educated upto Matriculation and above. On the contrary the Opioid group had an equal distribution of $50 \%$ below and above Matriculation.

Almost $65 \%$ of the Cannabinoid Group was either never employed or was presently unemployed due to their drug abuse or comorbid psychiatric disorders. The alcohol group on the other hand had $77 \%$ patients who were currently employed. Similar trends were seen in marital status with Alcohol group and other SUDs more likely to get married as compared to Opioid and Cannabinoid group. The other SUDs group had adolescents under age of 18 years and had therefore not attained the marriageable age. Cannabinoid group had $22 \%$ of cases who never got marriedeven after attaining marriageable 
age. This could again be due to severity of the drug abuse and severity of dual diagnosis cases which made them poor prognosis cases and were hence not able to find a life partner. Their personal income therefore was the lowest amongst all 4 groups. The significant Hindu majority in the study is more of a function of the societal majority of Hindus as a community in India.

Majority of the Opioid group patients were from urban areas which was significantly more than other groups which suggests that as far as West Bengal is concerned the Opioid addiction is more prevalent in urban areas than in rural areas whereas the Alcohol group had equal distribution as far as rural and urban distribution is concerned. The maximum number of patients coming to the Institute was from within $80 \mathrm{~km}$ radius which shows the referral radius of the De-addiction clinic of this institute. Majority of patients were referred to the Deaddiction Clinic from the general adult psychiatry clinic or from other departments of this tertiary superspeciality hospital.

The duration of use and duration of dependence in Alcohol group was 180 months and 100 months respectively suggesting that it took 7 years to develop dependence. On the contrary the duration of use and duration of dependence in Opioid group was 78 months and 62 months respectively. This suggests that Opioids produce dependence pattern much faster than any of the other 3 groups. The only exception to this is a small subgroup of Inhalant/ Solvent abusers who developed dependence as rapidly as did the Opioid group. The mean age of initiation of the various substancesin our study was interesting in the sense that Alcohol group had onset initiation in the early 30s where as the Cannabinoid and Opioid group had onset in the late teens or early 20s. Urban preponderance was seen in the Opioid group and Other SUDs (e.g. Volatile Solvent) unlike other groups where the distribution was more even suggesting that these type of patients are more prevalent in the urban population and have more treatment seeking behavior.
The Alcohol group had significantly more comorbid physical illnesses than other 3 groups. The Cannabinoid group (Group III) had significantly more comorbid Schizophrenia and schizophrenia like psychotic disorders where as Alcohol group (Group II) had significantly greater dual diagnosis of Bipolar Disorders and other affective disorders. Long standing dependence produced more physical illnesses in alcohol dependent. Cannabis dependence has high comorbidity with schizophrenia and there is well known entity of cannabis induced nonaffective psychosis. Cannabinoid group had high family history of SUD as compared to other groups. This further supports that there is genetic basis to the inheritance of these disorders. However, no significant difference was seen across groups on variable of Family history of Psychiatric Disorders.

In Pre-morbid personality, although there was no differences across groups howeverComorbid Cluster 'B'Personality disorders were seen in $26.5 \%$ of cases in opioid group. The study however could not find any increased chance of any group having any particular type of personality disorder.

Motivation to leave alcohol/drugs is an important indicator of repeated relapses and adverse outcome in cases of SUDs. Cannabinoid group had poorer motivation to leave drugs than other groups though the difference was non-significant. Another $50 \%$ of patients had superficial motivation. The presence of psychotic illness further decreases their motivation level.

Social support is an important factor in determination of outcome and treatment compliance as well as follow-ups and adherence to treatment. On comparison of Social Support no difference was found across groups with $80-95 \%$ patients having fair to good social support. This suggests that patients coming to our clinic had better social support. Also Indian patients like other groups of psychiatric disorders have a better outcome due to better social support. 
With increasing age there is increased Medical Illness suggesting that long duration of SUD caused various medical complications to develop in these patients. Age at presentation correlated with increase in Marital Complication and Social complications which meant that marital partners and the society at large had difficulty in coping with long duration of the illness. However those patients who were employed and were in the higher incomecategory were at a better chance of getting a life partner. Also those who got married were more likely to have well adjusted premorbid personality. But over the years these patients who did get the chance to get a life partner faced more marital complications.

Educational level correlated with greater social support. Higher income correlated positively with social status. Higher income correlated positively with increased Medical illness suggesting extravagant life style leads to drug/substance abuse as well as various life style medical illnesses. Income negatively correlated with Financial complications, family complications and social complications. Family income positively correlated with Duration of dependence suggesting that patients may have used their family resources to sustain their drug/ alcohol abuse. Poor social status meant greater financial complications. Comorbid Medical illness has a negative impact on marital status of these patients. Family history of substance abuse correlated with social support i.e. positive family history of substance abuse led to poor social support. Positive family history of psychiatric illness in first degree relatives correlated with marital problems possibly because it was difficult for life partners to deal with both their spouses alcohol/drug abuse and family's burden to deal with psychiatric illness in another family member.

Poor social support correlated with family complications, marital complications and social complications. Motivation clearly and significantly correlated with financial, family, marital and social complications suggesting that Motivation to leave alcohol/drugs is influenced by these factors. The motivation level influenced follow-up rates with poor to superficial motivation predicting a poorer follow up rates. On the other hand good and fair motivation correlated with increased adherence to attending Group therapy. Motivation to leave drugs/alcohol correlated with drop-out \& relapse rates with poorer outcomes in form of relapses and drop out related to poor motivation to leave drugs.

\section{CONCLUSIONS}

Psychiatric and medical comorbidities are frequent amongst SUD patients with adverse outcome like drop-out and relapse cases, so social support plays a definitive role in providing support and care to the relapsed cases. In our study Poor social support correlated with family complications, marital complications and social complications. Motivation clearly and significantly correlated with financial, family, marital and social complications suggesting that Motivation to leave alcohol/drugs is influenced by these factors. The motivation level influenced follow-up rates with poor to superficial motivation predicting a poorer follow up rates. On the other hand good and fair motivation correlated with increased adherence to attending Group therapy. Motivation to leave drugs/alcohol correlated with drop-out \& relapse rates with poorer outcomes in form of relapses and drop out related to poor motivation to leave drugs. Financial security in form of employment is critical for adherence to treatment and followup rates which in turn influences recovery and rehabilitation. Motivation is cornerstone to relapses and management of motivation enhancement and adherence to treatment can reduce relapses.

\section{REFERENCES}

Reddy MV and Chandrashekharan CR. Prevalence of mental and behavioural disorders in India : A meta-analysis. Indian J Psychiatry. 1998; 40 : 149-157.

Ray R. The extent, pattern and trends of drug abuse in India, National Survey, Ministry of Social Justice and Empowerment, Government of India and United Nations Office on Drugs And Crime, regional office for South Asia. 2004.

Drug Abuse Monitoring System (Ray 2004). 


\section{Socio-Demographic, Clinical Correlates and Predictors of Adverse Outcome in Substance Use Disorder}

Sadock BJ \& Sadock VA. Caplan and Sadock's Comprehensive text book of Psychiatry. $7^{\text {th }}$ edition. Lippincort Williams and Wilkins publishers; 2000 pp 942-949.

Kumar SM. Rapid assessment survey of drug abuse in India. Ministry of Social Justice and Empowerment, Government of India and United Nations office on drugs and crime (UNODC), Regional office for South Asia; 2011

Bansal RK \& Banerjee S. Substance use by child laborers. Indian J Psychiatry. 1993; 35 : 159-61.

Tripathi BM, Lal R. Substance abuse in children and adolescents. Indian J Pediatrics 1999; 66 : 569-575.

Benegal V, Satyaprakash M, Nagaraja D. Alcohol misuse in the Andaman and Nicobar Islands. Report on project commissioned by the Indian Council of Medical Research and funded by Action Aid, India. 2008

Ministry of Social Justice and Empowerment, Government of India, Author. "Annual reports, 1997-98, 1998-99, 1999-2000 and 2000-01". New Delhi : Ministry of Social Justice and Empowerment, Government of India.

Addiction Severity Index treatment planning manual. 2010

Winslow M, Ng WL, Mythily S et al. Socio-demographic profile and help-seeking behavior of buprenorphine abusers in Singapore. Ann Acad Med Singapore; 2006 (35) : 451-456.

Singh B, Singh V, Vij A. Sociodemographic profile of Substance abusers attending a De-addiction Centre in Gaziabad. Medico-legal Update; 2006 (6) : 13-15.
Mohan D, Desai NG. "Survey of drug dependence in the community, urban metropolis Delhi : survey resurvey". 1994. Report submitted to Indian Council of Medical Research (ICMR).

Chaturvedi HK, Mahanta J. Socio-cultural Diversity and substance use pattern in Arunachal Pradesh, India. Drug Alcohol Depend. 2004 Apr; 74(1) : 97-104.

Hazarika NC, Biswas D, Phukan RK, et al. prevalence and pattern of substance abuse at Banderdewa, a border area of Assam and Arunachal Pradesh. Indian J Psychiatry. 2000; 42 (3) : 262-266.

Chaturvedi HK, Phukan RK, Mahanta J. The association of selected sociodemographic factors and differences in patterns of substance use : a pilot study in selected areas of North East India. Subst Use Misuse. 2003 Jul; 38 (9) : 1305-1322.

Agarwal D, Lal A, Chandra R. Relapse Precipitants in alcohol addiction. Ind J Soc Res 2009 Mar; 6(1) : 80-83.

Varma VK, Singh A, Singh S, Malhotra AK. Extent and pattern of alcohol use in North India. Indian J Psychiatry. 1980; 22 :331-337.

Nessa A, Latif SA, Siddiqui NI, et al. Drug abuse and addiction. Mymensingh Med J. 2008 Jul; 17(2) : 227-235. 